\title{
KIỂM ĐỊNH THANG ĐO CHẤT LƯợNG GIÁO DỤC ĐẠI HỌC TÙ SỰ CẢM NHẬN CỦA SINH VIÊN NGẢNH QUẢN TR! KİNH DOANH TẠI TP. HỒ CHÍ MINH
}

\author{
ĐÀM TRÍ CUỜNG ${ }^{1}$, VÕ THI QUÝ ${ }^{2}$, PHẠM XUÂN LAN ${ }^{3}$ \\ ${ }^{1}$ Truoòng Đại học Công Nghiệp TP.HCM; \\ ${ }^{2}$ Trưòng Đại học Quốc tế - Đại học Quốc gia TP.HCM; \\ ${ }^{3}$ Trưòng Đại hoc Kinh tế TP.HCM; \\ damtricuong@iuh.edu.vn,vtquy@hcmiu.edu.vn,lanqtkd@ueh.edu.vn
}

Tóm tắt. Nghiên cứu này nhằm kiểm định thang đo chất lượng giáo dục đại học từ sự cảm nhận của sinh viên tại các trường đại học ở TP.HCM. Kết quả nghiên cứu cho thấy thang đo chất lượng giáo dục đại học từ sự cảm nhận của sinh viên gồm 6 thành phần thang đo: (1) chương trình đào tạo, (2) kỹ năng giảng dạy của giảng viền, (3) tương tác giữa giảng viên và sinh viên, (4) cơ sở vật chất, (5) tương tác doanh nghiệp - ngoại khóa, và (6) chất lượng dịch vụ hỗ trợ sinh viên. Trong các thành phần trên thì thành phần thang đo tương tác doanh nghiệp - ngoại khóa là thành phần thang đo mới được hình thành từ kết quả nghiên cứu, là thành phần thang đo được gộp từ thành phần thang đo tương tác giữa doanh nghiệp và nhà trường, và thang đo hoạt động ngoại khóa. Ngoài ra, nghiên cứu này cũng đề cập thang đo chất lượng dịch vụ hỗ trợ sinh viên mà các nghiên cứu trước chưa đề cập, là thang đo mới mà tác giả khám phá thông qua 3 cuộc thảo luận nhóm. Kết quả kiểm định bằng phương pháp phân tích nhân tố khẳng định $\mathrm{CFA}$ (Confirmatory Factor Analysis) cho thấy thang đo của các thành phần đã đạt yều cầu về độ tin cậy và giá trị (tính đơn hướng, giá trị hội tụ, giá trị phân biệt, độ tin cậy tổng hợp và phương sai trích). Cuối cùng, các tác giả cũng trình bày thảo luận kết quả nghiên cứu cũng như hạn chế và hướng nghiên cứu tiếp theo.

Từ khóa. Kiểm định thang đo, chất lượng dịch vụ, chất lượng giáo dục đại học, sinh viên, thành phố Hồ Chí Minh

\section{TESTING THE MEASURING SCALE OF HIGHER EDUCATION QUALITY FROM STUDENTS' PERSPECTIVE OF BUSINESS AND ADMINISTRATION SECTOR IN HO CHI MINH CITY}

\begin{abstract}
This study aims to test the measuring scale of higher education quality from the students' perspective at universities in Ho Chi Minh city. The study's results showed that the scale of higher education quality from students' perspective was explained by six components: (1) curriculum, (2) teaching skills of faculty, (3) interaction between faculty and students, (4) physical facilities, (5) businesses interaction - extracurricular activities, (6) students support services quality. In the above scale components, the business interaction - extracurricular activities scale is a new measuring scale from the research results, the scale of which is composed of the university-industry interaction scale and the extracurricular activities scale. In addition, this study also mentions the scale of student support services quality that previous studies did not mention, a new measureing scale that the author explored through three focus groups. The scale of the components above were tested by confirmatory factor analysis (CFA) showed that these scales met the requirements of reliability and validity (unidimensionality, convergent validity, discriminant validity, composite reliability and average variance extracted). Finally, the authors also discussed the research results as well as limitations and further research directions.

Keywords: Testing the measuring scale, service quality, higher education quality, students' perspective, Ho Chi Minh city
\end{abstract}




\section{GIỚI THIỆ}

Giáo dục đại học là rất quan trọng đối với sự phát triển của 1 quốc gia (Annamdevula và Bellamkonda, 2016) [2]. Mặt khác, xu hướng hội nhập quốc tế, thì vấn đề hội nhập giáo dục và sự giao thoa giữa các nền giáo dục trên thế giới đang diễn ra là rất phổ biến. Chen và cộng sự (2009) [7] cho rằng khi cạnh tranh quốc tế ngày càng trở nên gay gắt, nhiều quốc gia tăng cường đầu tư vào giáo dục đại học trong nỗ lực nâng cao sức cạnh tranh của họ. Để thích ứng với sự cạnh tranh rất lớn từ khắp nơi trên thế giới, có một yêu cầu quan trọng và ngay lập tức là cải thiện chất lượng giáo dục đại học nhằm đáp ứng xu hướng học thuật quốc tế và gia tăng tiêu chuẩn học thuật chung và chất lượng giáo dục. Bên cạnh đó, Lawrence và McCollough (2001) [21] cho rằng để đáp ứng mối quan tâm ngày càng tăng từ các bên liên quan đến trường đại học về chất lượng kém hay không đồng nhất thì các cơ sở giáo dục đại học phải gia tăng tìm cách cải thiện chất lượng giáo dục. Hiện nay tại TP. Hồ Chí Minh, trong thị trường giáo dục có tính cạnh tranh thì sinh viên được xem như là khách hàng. Hơn nữa, sinh viên phải trả tiền cho việc giáo dục cũng như tìm kiếm các trường đại học có chất lượng giáo dục cao để làm cơ sở cho việc chọn trường thì việc cải thiện và nâng cao chất lượng giáo dục là rất quan trọng đối với các trường đại học trong việc phục vụ và thu hút sinh viên.

Ngoài ra, các nhà quản lý giáo dục nhận thấy là để xây dựng thành công việc nâng cao chất lượng giáo dục đại học như là lợi thế cạnh tranh, trước tiên họ cần phải xác định được chất lượng giáo dục đại học bao gồm những thành phần nào. Dựa vào kết quả nghiên cứu định tính thông qua 3 cuộc thảo luận nhóm cho thấy thang đo chất lượng giáo dục đại học từ sự cảm nhận của sinh viên bao gồm 25 biến quan sát để đo lường 6 thành phần của chất lượng giáo dục đại học, đó là: (1) chương trình đào tạo, (2) giảng viên, (3) cơ sở vật chất, (4) tương tác giữa nhà trường và doanh nghiệp, (5) hoạt động ngoại khóa, và (6) chất lượng dịch vụ hỗ trợ sinh viên.

Do đó, mục tiêu của bài viết này là nhằm kiểm định thang đo chất lượng giáo dục đại học từ sự cảm nhận của sinh viên ngành quản trị kinh doanh tại các trường đại học ở TP.HCM.

Trong bài viết này, tác giả dựa vào cách tiếp cận giáo dục đại học là 1 dịch vụ, xem Trường học là đơn vị cung cấp dịch vụ và sinh viên là những người sử dụng dịch vụ. Mặt khác, trong bài viết này tác giả không tiếp cận đánh giá chất lượng của Trường học, không tiếp cận đánh giá chất lượng của chương trình đào tạo, mà ở đây tác giả chỉ tiếp cận đánh giá chất lượng cảm nhận của sinh viên (người sử dụng dịch vụ) về dịch vụ chất lượng giáo dục đại học mà họ sử dụng được cung cấp bởi Trường học. Ngoài ra, bài viết này không nghiên cứu đặc thù ngành $\mathrm{QTKD}$, mà giới hạn thị trường nghiên cứu là ngành $\mathrm{QTKD}$.

\section{CƠ SỞ LÝ THUYẾT VÀ THANG ĐO CHẤT LƯợNG GIÁO DỤC ĐẠI HỌC TỪ SỰ CẢM NHẬn CỦA SINH VIÊN}

\subsection{Cơ sở lý thuyết}

\subsubsection{Chất lượng dịch vụ}

Khái niệm chất lượng theo Annamdevula và Bellamkonda (2016) [2] là hiện tượng được thiết lập trong lý thuyết về quản lý, được đề xuất đầu tiên bởi Juran (1974) [19] và Deming (1982) [10]. Tuy nhiên, khái niệm chất lượng dịch vụ là khái niệm tương đối mới được giới thiệu bởi Gronroos (1984) [14] và Parasuraman và cộng sự (1998) [25]. Trong hơn 20 qua chất lượng dịch vụ đã được các nhà nghiên cứu thảo luận rất nhiều và nó trở thành một khái niệm đã được thiết lập do các yêu cầu kinh doanh toàn cầu năng động. Không giống như sản phẩm, dịch vụ có một số đặc điểm riêng biệt gây khó khăn cho việc đánh giá chất lượng của chúng. Đặc điểm nổi bật của dịch vụ là mang tính chất vô hình, nghĩa là nó không nhìn thấy, tiểp xúc hoặc tồn trữ mà chúng chỉ có thể cảm nhận và trải nghiệm (Gupta và Kaushik, 2018) [15]. Hiện nay, khái niệm chất lượng dịch vụ vẫn còn có sự khác nhau giữa các nhà nghiên cứu. Chẳng hạn: Chất lượng dịch vụ là khoảng cách giữa sự mong đợi về dịch vụ của khách hàng và cảm nhận của họ về kết quả của dịch vụ (xem Parasuraman và cộng sự, 1988 [25]; Lewis và Booms, 1983 [20]; Asubonteng và cộng sự, 1996 [3]; Gefen, 2002 [13], Gronroos, 1984 [14]). Chất lượng dịch vụ chỉ là cảm nhận của khách hàng về kết quả của dịch vụ (Cronin và Taylor, 1992) [9]. Chất lượng dịch vụ là sự kết hợp của hai từ dịch vụ và chất lượng mà chúng ta tìm thấy nhấn mạnh về sự sã̃n có của chất lượng dịch vụ cho người sử dụng cuối cùng và tập trung vào các tiêu chuẩn hoặc đặc điểm kỹ thuật mà một nhà cung cấp dịch vụ hứa hẹn (Prasad và Jha 2013) [27]. Chất lượng dịch vụ là thể hiện qua 7 đặc tính. Những đặc 
tính này là: (1) Đảm bảo: sự tin tưởng cũng như an toàn vật chất; (2) Tính nhất quán: nhận được cùng đối xử như nhau cho mỗi lần giao dịch; (3) Thái độ: lịch sự; (4) Đầy đủ: sự sẵn có của các dịch vụ phụ trợ; (5) Điều kiện: của cơ sở vật chất; (6) Sự sẵn có: không gian và thời gian khách hàng tiếp xúc với dịch vụ;

(7) Đào tạo: của những nhà cung cấp dịch vụ (Sasser và cộng sự 1978) [28].

Tóm lại, chất lượng dịch vụ là một khái niệm có nhiều quan điểm khác nhau. Tuy nhiên, có hai quan điểm về chất lượng dịch vụ thường được các nhà nghiên cứu đề cập đó là: (1) nó là cảm nhận của khách hàng về kết quả dịch vụ; (2) nó là khoảng cách giữa sự mong đợi về dịch vụ của khách hàng và cảm nhận của họ về kết quả của dịch vụ.

\subsubsection{Chất lượng giáo dục đại học}

Định nghĩa chất lượng giáo dục đại học được chứng minh là nhiệm vụ khó khăn và chưa có định nghĩa nhất quán (Cheng và Tam, 1997 [6]; Nguyễn Văn Tuấn, 2011 [23]). Các nhà nghiên cứu hàn lâm trên thế giới đưa ra những quan điểm khác nhau về định nghĩa chất lượng giáo dục đại học. Chẳng hạn, Harvey và Green (1993) [17] cho rằng chất lượng giáo dục đại học có thể tập hợp thành 5 nhóm quan điểm về chất lượng như sau: (1) chất lượng là sự vượt trội, (2) chất lượng là sự hoàn hảo, (3) chất lượng là phù hợp với mục tiêu, (4) chất lượng là giá trị đồng tiền và (5) chất lượng là sự chuyển đổi. Cheng (1996) [8] đề nghị rằng chất lượng giáo dục đại học là tập hợp các yếu tố đầu vào, quy trình và đầu ra của hệ thống đào tạo cung cấp dịch vụ hoàn toàn thỏa mãn cả những khách hàng chiến lược bên trong và bên ngoài bằng cách đáp ứng những mong đợi hiện tại và tiềm ẩn của họ. Cheng và Tam (1997) [6] cho rằng, nếu chúng ta chấp nhận định nghĩa này thì chất lượng giáo dục đại học là một khái niệm đa hướng. Harvey và Knight (1996) [19] giống như quan điểm của Harvey và Green (1993) [17] cho rằng chất lượng giáo dục đại học có thể tập hợp thành 5 nhóm quan điểm về chất lượng như sau: (1) Chất lượng là sự vượt trội, (2) Chất lượng là sự hoàn hảo, (3) Chất lượng là phù hợp với mục tiêu, (4) Chất lượng là giá trị đồng tiền, (5) Chất lượng là sự chuyển đổi. Parri (2006) [26]cho rằng chất lượng giáo dục đại học, có thể tập hợp thành các quan điểm chất lượng như sau: (1) Chất lượng là sự vượt trội, xuất sắc; (2) Chất lượng là không có lỗi; (3) Chất lượng là phù hợp với mục tiêu; (4) Chất lượng là sự chuyển đổi, định hình lại; (5) Chất lượng là ngưỡng tiêu chuẩn; (6) Chất lượng là sự nâng cao hay cải tiến; (7) Chất lượng là giá trị đồng tiền. Bộ Giáo dục và Đào tạo (2007) [4] cho rằng chất lượng giáo dục trường đại học là sự đáp ứng mục tiêu do trường đề ra, đảm bảo các yêu cầu về mục tiêu giáo dục đại học của Luật Giáo dục, phù hợp với yêu cầu đào tạo nguồn nhân lực cho sự phát triển kinh tế - xã hội của địa phương và cả nước. Nguyễn Văn Tuấn (2011) [23] theo các chuyên gia đầu ngành về chất lượng giáo dục, chất lượng có thể được nhìn nhận qua 5 khía cạnh: (1) Chất lượng được ngầm hiểu là chuẩn mực cao; (2) Chất lượng đề cập đến sự nhất quán và không sai sót trong việc thực thi một công tác giáo dục và đào tạo; (3) Chất lượng là hoàn tất những mục tiêu đề ra trong kế hoạch của nhà trường; (4) Chất lượng là những đo lường phản ánh thành quả thu được xứng đáng với đầu tư (hay nói nôm na là xứng đáng với "đồng tiền bát gạo"); (5) Chất lượng là một quy trình liên tục để "khách hàng" (tức sinh viên) có thể đánh giá sự hài lòng của họ khi theo học. Nguyễn Văn Tuấn (2011) [23] cho rằng chất lượng giáo dục đại học là tập hợp một số yếu tố liên quan đến đầu vào, quy trình đào tạo và đầu ra. UNESCO - United Nations Educational Scientific and Cultural Organization (Tổ chức Giáo dục, Khoa học và Văn hóa của Liên Hiệp Quốc) (1998) [29] cho rằng chất lượng giáo dục đại học là khái niệm đa hướng, bao gồm tất cả các chức năng và hoạt động như chương trình đào tạo, chất lượng giảng dạy giảng viên, nghiên cứu và học bổng, đặc điểm sinh viên, nhân viên, cơ sở vật chất, và môi trường học thuật. Chen và cộng sự (2007) [5] dựa vào định nghĩa của UNESCO (1998) [29], trên cơ sở đó các tác giả đưa ra định nghĩa chất lượng giáo dục đại học như sau: chất lượng giáo dục đại học là một khái niệm đa hướng bao gồm các chức năng và hoạt động như chương trình đào tạo, chất lượng giảng viên giảng dạy, chính phủ, cơ sở vật chất, đặc điểm sỉnh viên, quản lý và hành chính và hệ thống tương tác. O’Neill và Palmer (2004) [24] cho rằng chất lượng giáo dục đại học là sự khác biệt giữa những gì mà sinh viên mong muốn nhận được và nhận thức của họ về chuyển giao thực tế.

Tóm lại, chất lượng giáo dục đại học là một khái niệm phức tạp và đa hướng, một định nghĩa phù hợp duy nhất về chất lượng giáo dục đại học vẫn còn thiếu (Harvey và Green, 1993) [17]. Trong nghiên cứu này, định nghĩa chất lượng giáo dục đại học của O'Neill và Palmer (2004) [24] được sử dụng vì nó liên quan đến cảm nhận của sinh viên về chất lượng giáo dục. 


\subsection{Thang đo chất lượng giáo dục đại học}

Thang đo chất lượng giáo dục đại học từ sự cảm nhận của sinh viên được hình thành từ kết quả của các nghiên cứu trước cũng như được khám phá, điều chỉnh và bổ sung thông qua nghiên cứu định tính bằng phương pháp thảo luận nhóm. Để có được thang đo chất lượng giáo dục đại học từ sự cảm nhận của sinh viên, nhóm tác giả đã tiến hình 3 cuộc thảo luận nhóm với sinh viên. Lý do tiến hành 3 cuộc thảo luận nhóm là vì trong lần thảo luận nhóm lần 1 , chúng tôi chỉ tập trung vào khám phá các thành phần tạo nên chất lượng giáo dục đại học, và trong lần thảo luận nhóm lần 2 chúng tôi tập trung xem xét lại có phát hiện thêm thành phần mới nào nữa không? Do đó, trong 2 lần thảo luận nhóm lần 1 và lần 2 chúng tôi chỉ ghi nhận lại một cách cơ bản thang đo cho từng thành phần tạo nên chất lượng giáo dục đại học. Trong thảo luận nhóm lần 3 , bên cạnh chúng tôi tiêp tục khẳng định lại các thành phần tạo nên chất lượng giáo dục đại học, chúng tôi còn khám phá, điều chỉnh và bổ sung các thang đo các thành phần tạo nên chất lượng giáo dục đại học cho phù hợp với thị trường TP. Hồ Chí Minh.

Sinh viên được chọn tham gia thảo luận nhóm chủ yếu là những sinh viên năm 3 hoặc năm 4 . Đây là những sinh viên đã có trải nghiệm học tập tại trường đại học, do đó họ sẽ có cảm nhận chính xác về chất lượng giáo dục đại học được cung cấp bởi nhà trường.

Thang đo chất lượng giáo dục đại học từ sự cảm nhận của sinh viên được thể hiện qua 6 thành phần thang đo: (1) thang đo chương trình đào tạo được thể hiện qua 4 biến quan sát là CTDT1-Kiến thức nhà trường trang bị cho sinh viên đáp ứng nhu cầu thực tế, CTDT2-Nhà trường giải thích mục tiêu chương trình đào tạo cho sinh viên, CTDT3-Nội dung môn học giúp sinh viên phát triển các kỹ năng có thể áp dụng trong công việc thực tế, CTDT4-Kiến thức và kỹ năng thu được trong các môn học giúp sinh viên tìm được công việc phù hợp; (2) thang đo giảng viên gồm 2 thành phần là (2.1) thành phần kỹ năng giảng dạy của giảng viên gồm 4 biến quan sát: GD1-Giảng viên giảng dạy rất dễ hiểu, GD2-Giảng viên truyền đạt rõ ràng nội dung giảng dạy, GD3-Giảng viên chuẩn bị bài giảng kỹ, GD4-Giảng viên sử dụng hiệu quả thiết bị hồ trợ giảng dạy; và (2.2) thành phần tương tác giữa giảng viên và sinh viên gồm 3 biến quan sát: TT1-Giảng viên khuyến khích sinh viên thảo luận trong lớp, TT2-Giảng viên tạo cơ hội cho sinh viên đặt câu hỏi trong lớp, TT3-Giảng viên khuyến khích sinh viên phát biểu trong giờ học; (3) thành phần cơ sở vật chất gồm 5 biến quan sát: CSVC1- Phòng họp sạch sẽ, rộng rãi và thông thoáng, CSVC2-Phương tiện giảng dạy (máy chiếu, micro ...) có thể áp dụng trong việc dạy học hiện đại, CSVC3-Thư viện cung cấp đầy đủ tài liệu học tập và nghiên cứu cho sinh viên, CSVC4-Sinh viên có thể truy cập internet tại bất kỳ đâu trong khuôn viên trường, CSVC5-Cơ sở dữ liệu điện tử (tài liệu học tập, dữ liệu nghiên cứu ...) phục vụ tốt cho việc học tập; (4) thang đo tương tác giữa nhà trường và doanh nghiệp gồm 3 biến quan sát: TTDN1-Nhà trường tổ chức cho sinh viên những chuyến đi thực tế tại doanh nghiệp, TTDN2-Nhà trường mời diễn giả từ doanh nghiệp trao đổi, chia sẻ tình hình thực tế tại doanh nghiệp, TTDN3-Nhà trường giới thiệu đơn vị thực tập cho sinh viên; (5) thang đo hoạt động ngoại khóa gồm 3 biến quan sát: HDNK1-Nhà trường có hoạt động ngoại khóa (bao gồm các câu lạc bộ và các sự kiện xã hội) hữu ích cho sinh viên, HDNK2-Sinh viên có được những kỹ năng sống hữu ích từ hoạt động ngoại khóa, HDNK3Hoạt động ngoại khóa làm cho việc học của sinh viên thú vị hơn; và (6) thang đo chất lượng dịch vụ hỗ trợ sinh viên gồm 3 biến quan sát: HTSV1-Nhân viên khoa/phòng/ban làm việc khoa học, HTSV2-Nhân viên khoa/phòng/ban làm việc tận tâm với sinh viên, HTSV3-Nhân viên khoa/phòng/ban thân thiện với sinh viên. Trong 6 thành phần thang đo trên thì thành phần thang đo chất lượng dịch vụ hỗ trợ sinh viên là thang đo mới do tác giả khám phá thông qua 3 cuộc thảo luận nhóm (Đàm Trí Cường và Võ Thị Quý, 2015) [11].

\section{PHƯƠNG PHÁP NGHIÊN CÚU}

Bài viết này thực hiện nghiên cứu định lượng thông qua nghiên cứu định lượng sơ bộ và nghiên cứu định lượng chính thức.

Nghiên cứu định lượng sơ bộ được thực hiện bằng phương pháp phỏng vấn trực tiếp sinh viên của trường Đại học Kinh tế TPHCM thông qua bảng câu hỏi chi tiết sau khi đã hướng dẫn cách trả lời bảng câu hỏi. Nghĩa là tác giả trực tiếp trực tiểp đến lớp học của sinh viên ngành QTKD phỏng vấn sau khi đã hướng dẫn cách trả lời bảng câu hỏi. Mẫu cho nghiên cứu định lượng này có kích thước $\mathrm{n}=121$. Để có được kích thước mẫu này, tác giả phát ra 150 bảng câu hỏi và thu về 138 bảng câu hỏi. Có 17 bảng câu hỏi không phù hợp nên bị loại. Dữ liệu thu thập từ nghiên cứu này được sử dụng để đánh giá sơ bộ thang 
đo các khái niệm nghiên cứu về giá trị và hệ số tin cậy thang đo trước khi tiến hành nghiên cứu chính thức. Hai phương pháp được sử dụng là phương pháp phân tích nhân tố khám phá EFA (Exploratory Factor Analysis) và phương pháp hệ số tin cậy Cronbach alpha.

Nghiên cứu định lượng chính thức được thực hiện bằng cách phỏng vấn trực tiếp sinh viên các trường đại học tại TP.HCM gồm Đại học Kinh tế TP.HCM, Đại học Mở TP.HCM và Đại học Văn Lang. Trong mô hình thang đo các khái niệm này có 59 tham số cần ước lượng. Do đó, kích thước mẫu tối thiểu cần thiết cho nghiên cứu chính thức này là $\mathrm{n}=295$ (59x5). Tuy nhiên, trong nghiên này kích thước mẫu của nghiên cứu này là $n=2713$. Lý do của việc lấy mẫu khảo sát lớn (2713 sinh viên) là nhằm kiểm tra tính ổn định của thang đo. Mục tiêu chính của nghiên cứu này là kiểm định lại thang đo thông qua phương pháp nhân tố khẳng định CFA (Confirmatory Factor Analysis). Thang đo các thành phần được kiểm định lại tính đơn hướng, giá trị hội tụ, giá trị phân biệt, độ tin cậy tổng hợp và phương sai trích. Đối với nghiên cứu có kích thước mẫu lớn thì mô hình nghiên cứu được xem là phù hợp với dữ liệu thị trường khi nhận được các giá trị GFI, TLI, CFI từ .90 đến 1; RMSEA $\leq .08$ (Nguyễn Đình Thọ và Nguyễn Thị Mai Trang, 2008 [22]). Ngoài các chỉ tiêu trên, kết quả kiểm định cũng phải đảm bảo độ tin cậy tổng hợp > .60; phương sai trích phải lớn hơn .50 (Hair và cộng sự, 1998 [16]).

\section{KẾT QUẢ NGHIÊN CÚU}

\subsection{Kết quả nghiên cứu định lượng sơ bộ}

\subsubsection{Kết quả phân tích EFA - Đánh giá giá trị thang đo}

Kết quả phân tích EFA cho thấy có 7 nhân tố trích tại eigenvalue $=1.037>1$ và có tổng phương sai trích được là $73.37 \%>50 \%$ (xem Bảng 1 ). Nhân tố 1 - chương trình đào tạo (CTDT) có trọng số nhân tố nhỏ nhất là .692 > .50. Nhân tố $2-$ chất lượng dịch vụ hỗ trợ sinh viên (HTSV) có trọng số nhân tố nhỏ nhất là $.846>.50$. Nhân tố $3-$ cơ sở vật chất $(\mathrm{CSVC})$ có trọng số nhân tố nhỏ nhất là $.539>.50$. Nhân tố 4 - kỹ năng giảng dạy của giảng viên (GD) có trọng số nhân tố nhỏ nhất là .677> .50. Nhân tố 5 - tương tác giữa nhà trường và doanh nghiệp (TTDN) có trọng số nhân tố nhỏ nhất là $.619>.50$. Nhân tố 6 - hoạt động ngoại khóa (HDNK) có trọng số nhân tố nhỏ nhất là $.705>.50$. Nhân tố 7 - tương tác giữa giảng viên và sinh viên (TTSV) có trọng số nhân tố nhỏ nhất là $.650>.50$. Và có chêch lệch trọng số nhân tố giữa trọng số nhân tố của biến đó nhóm vào so với các nhân tố khác đều $>.30$. Do đó, giá trị thang đo các khái niệm này đạt yêu cầu. Tuy nhiên, thang đo giảng viên theo kết quả nghiên cứu định tính thông qua thảo luận nhóm là thang đo đa hướng gồm 2 thành phần là kỹ năng giảng dạy của giảng viên, và tương tác giữa giảng viên và sinh viên nhưng khi phân tích EFA chung các thang đo đơn hướng và đa hướng thì kết quả EFA cho thấy các thành phần của thang đo giảng viên lại trở thành các thang đo đơn hướng. Do đó, phân tích nhân tố khẳng định CFA (Confirmatory Factor Analysis) tiếp theo sẽ kiểm định lại kết quả này.

\subsubsection{Kết quả Cronbach's alpha - Đánh giá độ tin cậy của thang đo}

Kết quả Cronbach's alpha cho thấy các thang đo đều đạt độ tin cậy. Các hệ số tương quan biến-tổng đều cao (nhỏ nhất là biến CSVC2 $=.499>.30$ ). Cronbach's alpha của các thang đo đều cao, nhỏ nhất là thang đo kỹ năng giảng dạy của giảng viên $(.772>.70)$.

Cụ thể là Cronbach's alpha của thang đo chương trình đào tạo (CTDT) là .885; của thang đo chất lượng dịch vụ hỗ trợ sinh viên (HTSV) là .921; của thang đo cơ sở vật chất (CSVC) là .828; của thang đo kỹ năng giảng dạy của giảng viên (GD) là .772; của thang đo tương tác giữa nhà trường và doanh nghiệp (TTDN) là .773; của thang đo hoạt động ngoại khóa (HDNK) là .795; của thang đo tương tác giữa giảng viên và sinh viên (TTSV) là .794 (xem Bảng 1 ). 
Bảng 1: Kết quả phân tích EFA và Cronbach alpha các thang đo

\begin{tabular}{|c|c|c|c|c|c|c|c|}
\hline & \multicolumn{7}{|c|}{ Nhân tố } \\
\hline & 1 & 2 & 3 & 4 & 5 & 6 & 7 \\
\hline CTDT3 & .915 & & & & & & \\
\hline CTDT4 & .832 & & & & & & \\
\hline CTDT1 & .818 & & & & & & \\
\hline CTDT2 & .692 & & & & & & \\
\hline HTSV2 & & .882 & & & & & \\
\hline HTSV1 & & .849 & & & & & \\
\hline HTSV3 & & .846 & & & & & \\
\hline CSVC1 & & & .785 & & & & \\
\hline CSVC4 & & & .645 & & & & \\
\hline CSVC2 & & & 639 & & & & \\
\hline CSVC5 & & & .629 & & & & \\
\hline CSVC3 & & & .539 & & & & \\
\hline GD2 & & & & .753 & & & \\
\hline GD3 & & & & .682 & & & \\
\hline GD1 & & & & .680 & & & \\
\hline GD4 & & & & .677 & & & \\
\hline TTDN2 & & & & & .832 & & \\
\hline TTDN1 & & & & & .768 & & \\
\hline TTDN3 & & & & & .619 & & \\
\hline HDNK2 & & & & & & .852 & \\
\hline HDNK3 & & & & & & .832 & \\
\hline HDNK1 & & & & & & .705 & \\
\hline TTSV2 & & & & & & & .857 \\
\hline TTSV3 & & & & & & & .701 \\
\hline TTSV1 & & & & & & & .650 \\
\hline Eigenvalue & 8.076 & 2.659 & 2.228 & 1.739 & 1.377 & 1.226 & 1.037 \\
\hline Phương sai trích $(\%)$ & 12.529 & 11.976 & 11.204 & 9.983 & 9.502 & 9.431 & 8.742 \\
\hline Cronbach alpha & .885 & .921 & .828 & .772 & .773 & .795 & .794 \\
\hline
\end{tabular}

\subsection{Kết quả nghiên cứu chính thức}

\subsubsection{Kết quả phân tích EFA tại 3 trường đại học}

Kết quả phân tích EFA lần 1, có 3 biến quan sát bị loại (GD4, CSVC4 và HDNK3) vì có trọng số nhân tố $<.50$. Kết quả phân tích EFA lần 2 cho thấy có 6 nhân tố trích tại eigenvalue $=1.103$ và tổng phương sai trích được là $70.15 \%>.50 \%$ (Bảng 2). Các nhân tố đều có trọng số nhân tố > .50. Và có chêch lệch trọng số nhân tố giữa trọng số nhân tố của biến đó nhóm vào so các nhân tố khác đều > .30. Do đó, giá trị thang đo các khái niệm này đạt yêu cầu. Tuy nhiên, thang đo giảng viên theo kết quả nghiên cứu định tính thông qua thảo luận nhóm là thang đo đa hướng gồm 2 thành phần là kỹ năng giảng dạy của giảng viên, và tương tác giữa giảng viên và sinh viên nhưng khi phân tích EFA chung các thang đo đơn hướng và đa hướng thì kết quả EFA tại 3 trường đại học cho thấy các thành phần của thang đo giảng viên lại trở thành các thang đo đơn hướng. Ngoài ra, thang đo tương tác giữa nhà trường và doanh nghiệp, và thang đo hoạt động ngoại khóa được gộp lại thành 1 thang đo - thang đo này được đặt tên là thang đo tương tác doanh nghiệp - ngoại khóa. Do đó, phân tích CFA tiếp theo sẽ kiểm định lại kết quả này. 


\subsubsection{Kết quả Cronbach's alpha - Đánh giá độ tin cậy của thang đo tại 3 trường đại học}

Kết quả Cronbach's alpha cho thấy các thang đo đều đạt độ tin cậy. Các hệ số tương quan biến-tổng đều cao (nhỏ nhất là biến CSVC2 $=.417>.30$ ). Cronbach's alpha của các thang đo đều cao, nhỏ nhất là thang đo chương trình đào tạo $(.772>.70)$.

Cụ thể là Cronbach's alpha của thang đo cơ sở vật chất (CSVC) là .812; của thang đo tương tác doanh nghiệp - ngoại khóa (TTDN-NK) là .832; của thang đo chất lượng dịch vụ hỗ trợ sinh viên (HTSV) là .924; của thang đo chương trình đào tạo (CTDT) là .772; của thang đo tương tác giữa giảng viên và sinh viên (TTSV) là .847; của thang đo kỹ năng giảng dạy của giảng viên (GD) là .846.

Bảng 2: Kết quả phân tích EFA các thang đo tại 3 trường đại học

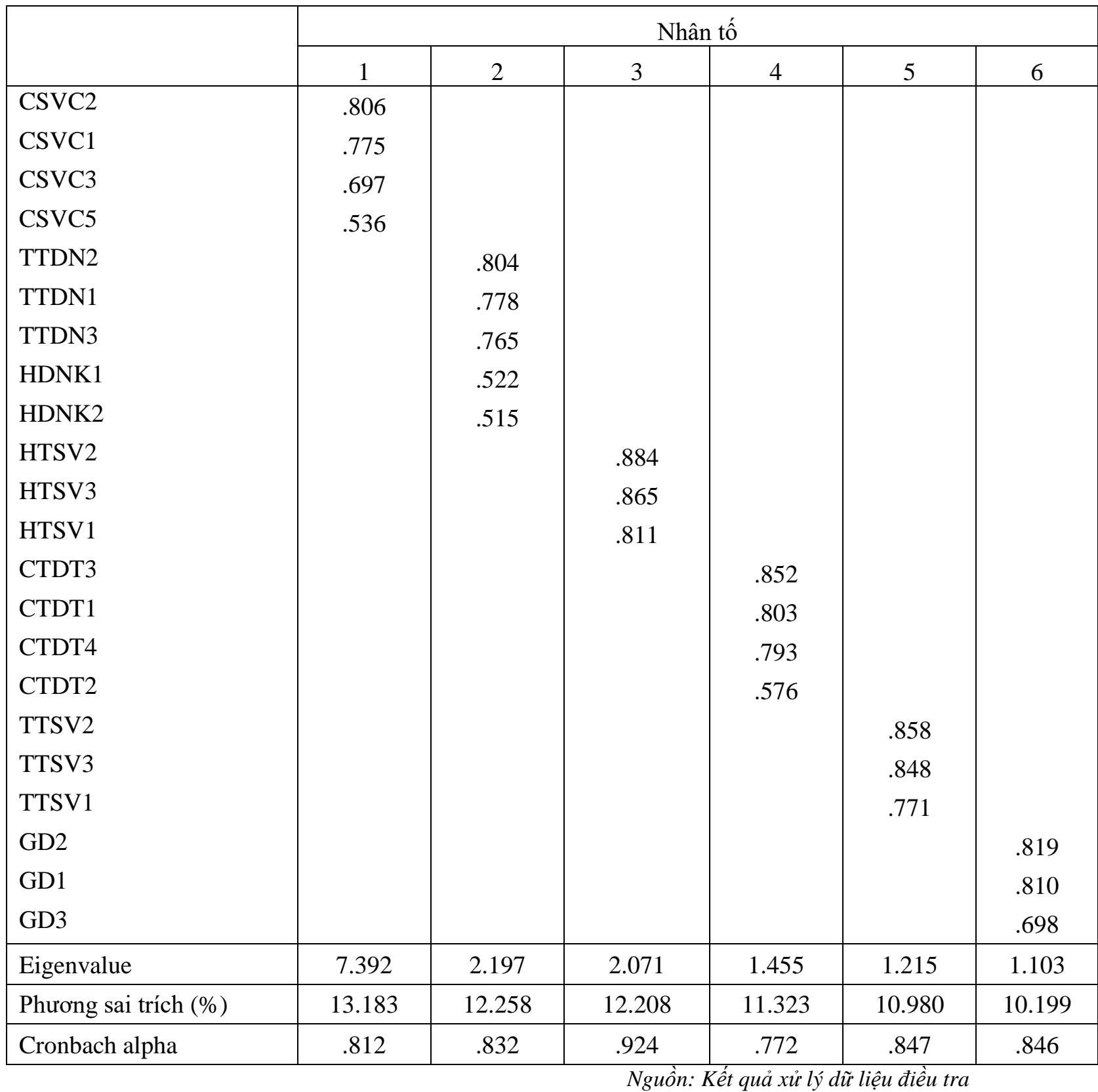

4.2.3. Kết quả phân tích CFA - kiểm định thang đo chất lượng giáo dục đại học từ sự cảm nhận của sinh viên tại 3 trường đại học 


\section{KIỂM ĐỊNH THANG ĐO CHẤT LƯợNG GIÁO DỤC ĐẠI HỌC TỬ SỰ CẢM NHẬN CỦA SINH VIÊN NGÀNH QUẢN TRI KINH DOANH TẠI TP. HỒ CHÍ MINH}

Kết quả phân tích CFA cho thấy các biến quan sát CTDT2, TTDN1 và TTDN3 bị loại vì có hệ số hồi quy chuẩn hóa thấp (không lớn hơn .50) (Anderson và Gerbring, 1998 [1]).

Sau khi loại các biến quan sát CTDT2, TTDN1 và TTDN3. Mô hình CFA được trình bày trong Hình 1.

Qua Hình 1 , kết quả phân tích CFA cho thấy mô hình này phù hợp với dữ liệu thị trường: Chi-square $=1344.652 ; \mathrm{df}=137 ; \mathrm{p}=.000 ; \mathrm{GFI}=.948 ; \mathrm{TLI}=.948 ; \mathrm{CFI}=.958$. Các giá trị GFI, TLI và $\mathrm{CFI}$ đều nằm trong khoản từ .90 đến $1 ;$ RMSEA $=.057 \leq .08$. Kết quả trên khẳng định tính đơn hướng của các thang đo: chương trình đào tạo; kỹ năng giảng dạy của giảng viên; tương tác giữa giảng viên và sinh viên; cơ sở vật chất; tương tác doanh nghiệp - ngoại khóa; và chất lượng dịch vụ hỗ trợ sinh viên. Bên cạnh đó, hệ số hệ quy chuẩn hóa của các biến quan sát đều lớn hơn .50 (nhỏ nhất là .54) và có ý nghĩa thống kê (tất cả giá trị $\mathrm{P}=.000<.05$ ), do đó, mô hình đạt giá trị hội tụ. Ngoài ra, mô hình không có tương quan giữa các cặp khái niệm vì có hệ số tương quan khác 1 và có ý nghĩa thống kê $(\mathrm{P}=.000<.05)$ nên các khái niệm đều đạt giá trị phân biệt.

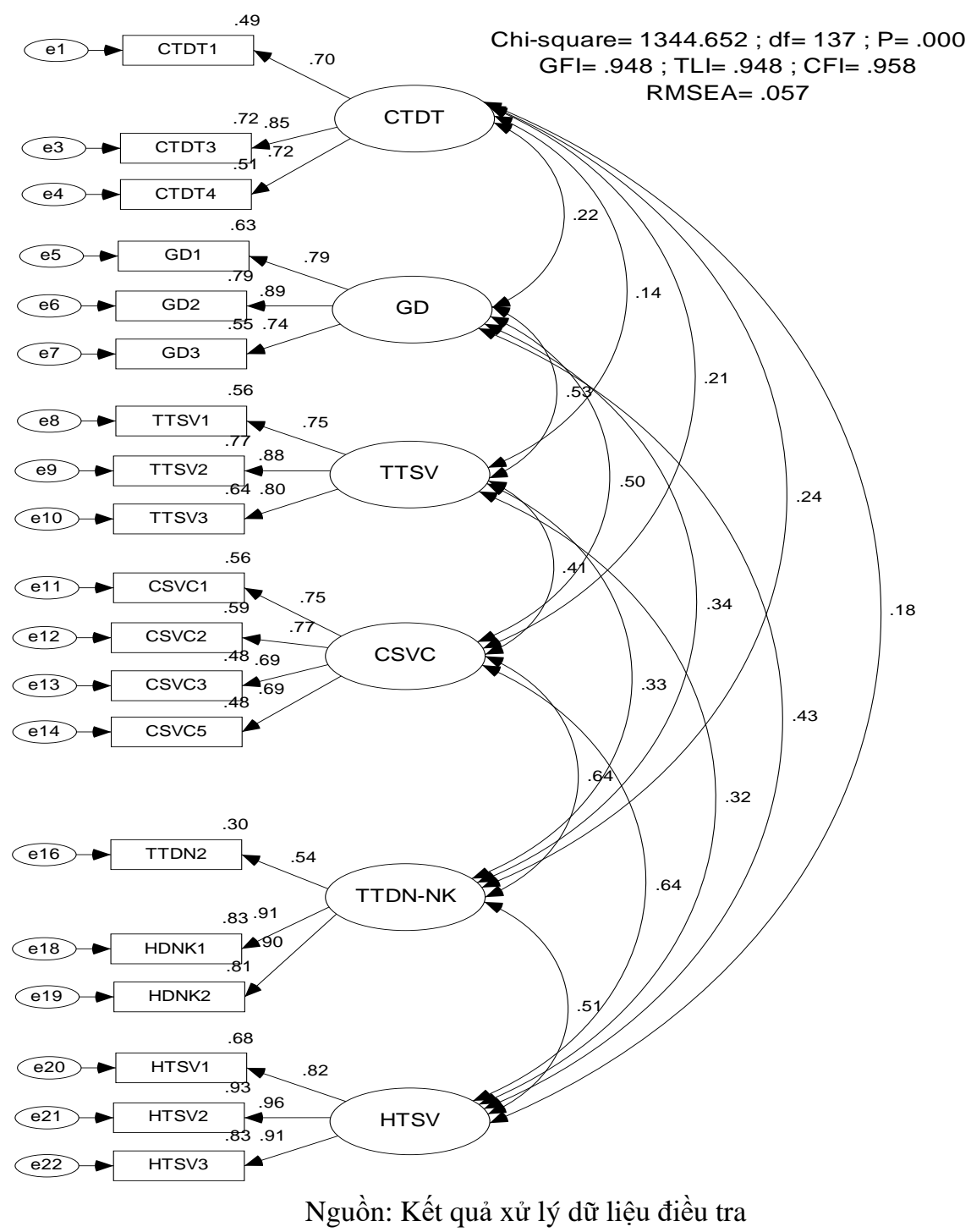

Hình 1: Kết quả phân tích CFA các thang đo tại 3 trường đại học

Hệ số tin cậy tổng hợp và phương sai trích của các khái niệm thể hiện qua Bảng 3. 
Bảng 3: Kết quả kiểm định hệ số tin cậy tổng hợp và phương sai trích của các khái niệm

\begin{tabular}{|l|c|c|}
\hline \multicolumn{1}{|c|}{ Khái niệm } & $\begin{array}{c}\text { Độ tin cậy tổng hộp } \\
\left(\rho_{\mathrm{c}}\right)\end{array}$ & $\begin{array}{c}\text { Phương sai trích } \\
\left(\rho_{\mathrm{vc}}\right)\end{array}$ \\
\hline Chương trình đào tạo & .873 & .6345 \\
\hline Kỹ năng giảng dạy của giảng viên & .911 & .6414 \\
\hline Tương tác giữa giảng viên và sinh viên & .911 & .6420 \\
\hline Cơ sở vật chất & .884 & .6324 \\
\hline Tương tác doanh nghiệp - ngoại khóa & .895 & .6228 \\
\hline Chất lượng dịch vụ hỗ trợ sinh viên & .960 & .6511 \\
\hline
\end{tabular}

Nguồn: Kết quả xủ lý dũ liệu điều tra

Qua Bảng 3 cho thấy thang đo của 6 khái niệm: chương trình đào tạo; kỹ năng giảng dạy của giảng viên; tương tác giữa giảng viên và sinh viên; cơ sở vật chất; tương tác doanh nghiệp - ngoại khóa; và chất lượng dịch vụ hỗ trợ sinh viên đều đạt yêu cầu về độ tin cậy tổng hợp, thấp nhất là .873 > .60 và phương sai trích, thấp nhất là $.6228>.50$.

Bảng 4 trình bày tóm tắt kết quả kiểm định thang đo các khái niệm.

Bảng 4: Tóm tắt kết quả kiểm định thang đo các khái niệm

\begin{tabular}{|l|c|c|c|c|c|c|}
\hline \multicolumn{1}{|c|}{ Khái niệm } & Ký hiệu & $\begin{array}{c}\text { Số biến } \\
\text { quan } \\
\text { sát }\end{array}$ & $\begin{array}{c}\text { Sự phù hợp } \\
\text { (đơn hướng, } \\
\text { hội tụ và } \\
\text { phân biệt) }\end{array}$ & $\begin{array}{c}\text { Độ tin } \\
\text { cậy } \\
\text { tông } \\
\text { hợp }\end{array}$ & $\begin{array}{c}\text { Phương sai } \\
\text { trích }\end{array}$ & $\begin{array}{c}\text { Kết quả } \\
\text { kiểm định }\end{array}$ \\
\hline Chương trình đào tạo & CTDT & 3 & Phù hợp & .873 & .6345 & Phù hợp \\
\hline $\begin{array}{l}\text { Kỹ năng giảng dạy của } \\
\text { giảng viên }\end{array}$ & GD & 3 & Phù hợp & .911 & .6414 & Phù hợp \\
\hline $\begin{array}{l}\text { Tương tác giữa giảng viên } \\
\text { và sinh viên }\end{array}$ & TTSV & 3 & Phù hợp & .911 & .6420 & Phù hợp \\
\hline Cơ sở vật chất & CSVC & 4 & Phù hợp & .884 & .6324 & Phù hợp \\
\hline $\begin{array}{l}\text { Tương tác doanh nghiệp - } \\
\text { ngoại khóa }\end{array}$ & TTDN-NK & 3 & Phù hợp & .895 & .6228 & Phù hợp \\
\hline $\begin{array}{l}\text { Chất lượng dịch vụ hỗ trợ } \\
\text { sinh viên }\end{array}$ & HTSV & 3 & Phù hợp & .960 & .6511 & Phù hợp \\
\hline
\end{tabular}

Nguồn: Kết quả xủ lý dũ liệu điều tra

\section{THẢO LUẬN KẾT QUẢ NGHIÊN CÚU}

Kết quả nghiên cứu cho thấy thang đo chất lượng giáo dục đại học từ sự cảm nhận của sinh viên gồm 6 thành phần thang đo có ý nghĩa: (1) chương trình đào tạo, (2) kỹ năng giảng dạy của giảng viên, (3) tương tác giữa giảng viên và sinh viên, (4) cơ sở vật chất, (5) tương tác doanh nghiệp - ngoại khóa và (6) chất lượng dịch vụ hỗ trợ sinh viên. Trong các thành phần trên thì thành phần thang đo tương tác doanh nghiệp - ngoại khóa là thành phần thang đo mới được hình thành từ kết quả nghiên cứu, là thành phần thang đo được gộp từ thành phần thang đo tương tác giữa doanh nghiệp và nhà trường, và thang đo hoạt động ngoại khóa. Ngoài ra, nghiên cứu này cũng đề cập thang đo chất lượng dịch vụ hỗ trợ sinh viên mà các nghiên cứu trước chưa đề cập, là thang đo mới mà tác giả khám phá thông qua 3 cuộc thảo luận nhóm. Kết quả kiểm định bằng phương pháp phân tích nhân tố khẳng định CFA (Confirmatory Factor Analysis) cho thấy thang đo của các thành phần đã đạt yều cầu về độ tin cậy và giá trị (tính đơn hướng, giá trị hội tụ, giá trị phân biệt, độ tin cậy tổng hợp và phương sai trích). 
Do đó, kết quả nghiên cứu và kết quả kiểm định cho chúng ta một số hàm ý về mặt lý thuyết và thực tiễn như sau:

Về mặt lý thuyết: nghiên cứu này sẽ góp phần làm phong phú thêm thang đo chất lượng giáo dục đại học. Đồng thời, nghiên cứu này bổ sung vào thang đo chất lượng giáo dục đại học từ sự cảm nhận của sinh viên. Cụ thể, nghiên cứu này bổ sung vào thang đo tương tác doanh nghiệp - ngoại khóa; và thang đo chất lượng dịch vụ hỗ trợ sinh viên. Điều này, có thể giúp cho các nhà nghiên cứu hàn lâm và ứng dụng trong lĩnh vực này tại Việt Nam có được hệ thống thang đo để điều chỉnh, bổ sung và sử dụng cho các nghiên cứu của mình tại thị trường Việt Nam.

Về mặt thực tiễn: các thang đo trên giúp cho những nhà quản lý giáo dục đại học có liên quan sử dụng và điều chỉnh để sinh viên đánh giá chất lượng giáo dục đại học tại các trường đại học Việt Nam. Mặt khác, kết quả nghiên cứu trên giúp các nhà quản lý giáo dục đại học biết được các thành phần quan trọng nhất tạo nên chất lượng giáo dục đại học ngành QTKD từ sự cảm nhận của sinh viên đó là: (1) chương trình đào tạo, (2) kỹ năng giảng dạy của giảng viên, (3) tương tác giữa giảng viên và sinh viên, (4) cơ sở vật chất, (5) tương tác doanh nghiệp - ngoại khóa, và (6) chất lượng dịch vụ hỗ trợ sinh viên. Những thành phần trên là những thành phần quyết định đến chất lượng giáo dục bậc đại học ngành QTKD. Trên cơ sở đó, giúp họ đề ra cách thức quản lý hợp lý trong lĩnh vực giáo dục đại học nhằm nâng cao chất lượng giáo dục đại học ngành QTKD.

\section{KÊT LUẬN, HẠN CHẾ VÀ HƯớNG NGHIÊN CÚU TIÊP THEO}

Bài viết này đã đạt được mục tiêu là kiểm định thang đo chất lượng giáo dục đại học từ sự cảm nhận của sinh viên. Kết quả kiểm định cho thấy thang đo chất lượng giáo dục đại học từ sự cảm nhận của sinh viên gồm có 6 thành phần đó là: (1) chương trình đào tạo; (2) kỹ năng giảng dạy của giảng viên; (3) tương tác giữa giảng viên và sinh viên; (4) cơ sở vật chất; (5) tương tác doanh nghiệp và ngoại khóa; và (6) chất lượng dịch vụ hỗ trợ sinh viên. Trong các thành phần trên thì thành phần thang đo tương tác doanh nghiệp - ngoại khóa là thành phần thang đo mới được hình thành từ kết quả nghiên cứu, là thành phần thang đo được gộp từ thành phần thang đo tương tác giữa doanh nghiệp và nhà trường, và thang đo hoạt động ngoại khóa. Ngoài ra, nghiên cứu này cũng đề cập thang đo chất lượng dịch vụ hỗ trợ sinh viên mà các nghiên cứu trước chưa đề cập, là thang đo mới mà tác giả khám phá thông qua 3 cuộc thảo luận nhóm. Thang đo cho mỗi thành phần đã được kiểm định lại bằng phân tích nhân tố khẳng định CFA cho thấy các thang đo đạt yêu cầu về độ tin cậy và giá trị (độ tin cậy tổng hợp, tính đơn hướng, phương sai trích, giá trị hội tụ và giá trị phân biệt).

Nghiên cứu này cũng có những hạn chế là (1) nghiên cứu này chỉ đề cập đến các thành phần chính tạo nên chất lượng giáo dục đại học từ sự cảm nhận của sinh viên. Còn có những cách nhìn khác về chất lượng giáo dục đại học như cách nhìn trên góc độ chính phủ, nhà quản lý, giảng viên, nhà tuyển dụng ... Những cách nhìn trên những góc độ khác nhau về chất lượng giáo dục đại học sẽ có những kết quả khác nhau về các thành phần tạo nên chất lượng giáo dục đại học; (2) nghiên cứu này không nghiên cứu đặc thù của ngành QTKD mà giới hạn thị trường nghiên cứu là ngành QTKD; (3) nghiên cứu này chỉ khám phá thang đo chất lượng giáo dục đại học ngành QTKD từ sự cảm nhận của sinh viên, chứ không nghiên cứu mối quan hệ (chẳng hạn: mối quan hệ giữa thang đo chất lượng giáo dục đại học với sự hài lòng sinh viên hay lòng trung thành của sinh viên ...). Những hạn chế trên mở ra hướng nghiên cứu tiểp theo.

\section{TÀI LIỆU THAM KHẢO}

[1] Anderson, P.F. and Gerbing, D.W. (1998). Structual equation modelling in practice: a review and recommended two-step approach. Psychological Bulletin, 103(3), pp.411-23.

[2] Annamdevula, S. and Bellamkonda (2016). Effect of student perceived service quality on student satisfaction, loyalty and motivation in Indian universities: development of HiEduQual. Journal of Modelling in Management, 11(2), pp.446-62.

[3] Asubonteng, P., McCleary, K.J. and Swan, J.E. (1996). SERVQUAL revisited: a critical review of service quality. The Journal of Services Marketing, 10(6), pp.62-81.

[4] Bộ Giáo dục và Đào tạo (2007). Quyết định số 65/2007/QĐ-BGDĐT - Quyết định Ban hành Quy định về tiêu chuẩn đánh giá chất lượng giáo dục trường đại học. Hà Nội.

[5] Chen, C.Y., Sok, P. and Sok, K. (2007). Benchmarking potential factors leading to 


\section{CẢM NHẬN CỦA SINH VIÊN NGÀNH QUẢN TRI KINH DOANH TẠI TP. HỒ CHÍ MINH}

education quality: A study of Cambodian higher education. Quality Assurance in Education, 15(2), pp.12848.

[6] Cheng, Y.C. and Tam, W.M. (1997). Multi-models of quality in education. Quality Assurance in Education, 5(1), pp.22-51.

[7] Chen, S.H., Wang, H.H. and Yang, K.J., 2009. Establishment and application of performance measure indicators for universities. The TQM Magazine, 21(3), pp.220-35.

[8] Cheng, Y.C. (1996). The pursuit of school effectiveness: Theory, policy and research. Hong Kong: The Hong Kong Institute of Educational Research, The Chinese University of Hong Kong.

[9] Cronin, J.J. and Taylor, S.A. (1992). Measuring service quality: a re-examination and extension. Journal of Marketing, 56(3), pp.55-68.

[10] Deming, W. E. (1982). Quality, productivity, and competitive position. Cambridge, MA: Massachusetts Institute of Technology, Center for Advanced Engineering Study.

[11] Đàm Trí Cường và Võ Thị Quý (2015). Khám phá các thành phần chất lượng đào tạo đại học trên góc độ sinh viên - Một nghiên cứu tại Việt Nam. Tạp chí Kinh tế và Phát triển, số 215(II), trang 133-39.

[12] Gamage, D.T., Suwanabroma, J., Ueyama, T., Hada, S. and Sekikawa, E. (2008). The impact of quality assurance measures on student services at the Japanese and Thai private universities. Quality Assurance in Education, 16(2), pp.181-98.

[13] Gefen, D., 2002. Customer loyalty in e-commerce. Journal of the Association for Information Systems, 3(1), pp.27-51.

[14] Gronroos, C. (1984). A service quality model and its marketing implications. European Journal of Marketing, 18(4), pp.36-44.

[15] Gupta, P. and Kaushik (2018). Dimensions of service quality in higher education - critical review (students' perspective). International Journal of Educational Management, 32(4), pp.580-605.

[16] Hair J.F., Anderson, R.E., Tatham, R.L., and Black, W.C. (1998), Multivariate data analysis with reading, 5th ed, Prentice-Hall, International, Inc

[17] Harvey, L. and Green, D. (1993). Defining quality. Assessment and Education in Higher Education, 18(1), pp.9-34.

[18] Harvey, L. and Knight, P.T. (1996). Transforming Higher Education. Buckingham: The SRHE and Open University Press.

[19] Juran, J.M. (1974). Quality Control Handbook, $3^{\text {rd }}$ ed. New York: McGraw-Hill.

[20] Lewis, R.C. and Booms, B.H. (1983). The marketing aspects of service quality. In Berry, L., Shostack, G. and Upah, G. (Eds), ed. Emerging Perspectives on Services Marketing. Chicago: American Marketing. pp.99-107.

[21] Lawrence, J.J. and McCollough, M.A., 2001. A conceptual framework for guaranteeing higher education. Quality Assurance in Education, 9(3), pp.139-52

[22] Nguyễn Đình Thọ và Nguyễn Thị Mai Trang (2008). Nghiên cứu khoa học Marketing: Úng dụng mô hình cấu trúc tuyến tính SEM. TP. Hồ Chí Minh: NXB Đại học Quốc Gia TP.HCM

[23] Nguyễn Văn Tuấn (2011). Chất luợng giáo dục đại học nhìn tù góc độ hội nhập. TP. Hồ Chí Minh: NXB Tổng Hợp TP. Hồ Chí Minh và Thời Báo Kinh Tế Sài Gòn.

[24] O'Neil, M.A. and Palmer, A. (2004). Importance-Performance analysis: A useful tool for directing continuous quality improvement in higher education. Quality Assurance in Education, 12(1), pp.39-52.

[25] Parasuraman, A., Zeithaml, V.A. and Berry, L.L. (1988). ERVQUAL: A multiple-item scale for measuring consumer perceptions of service quality. Journal of Retailing, 64(1), pp.12-40.

[26] Parri, J. (2006). Quality in higher education. Journal Vadyba/Management, 2(11), pp.107-11.

[27] Prasad, R.K. and Jha, M.K. (2013). Quality measures in higher education: A review and conceptual model. Journal of Research in Business and Management, 1(3), pp.23-40.

[28] Sasser, W.E., Olsen, R.P. and Wyckoff, D.D. (1978). Management of service operations. Boston, MA: Allyn \& Bacon.

[29] UNESCO (1998). Higher education in the twenty-first century: Vision and Action. In World Conference on Higher Education. Paris, 1998. 